\title{
Knowledge and Attitude Regarding Organ Donation Among Medical Students in Hong Kong - A Cross Sectional Study
}

\section{Steven Tsun-Wai Chu ( $\nabla$ stwchu@connect.hku.hk)}

University of Hong Kong

Phoebe Pui Wun Chung

University of Hong Kong

Hiu Wai Lam

University of Hong Kong

Yau Long Hui

University of Hong Kong

Hing Chung Choi

University of Hong Kong

Ling Ling Sin

University of Hong Kong

Chui Shan Law

University of Hong Kong

Nga Ying Yan

University of Hong Kong

Ka Yung Choi

University of Hong Kong

Eric Yuk Fai Wan

University of Hong Kong

\section{Research Article}

Keywords: Organ donation, Knowledge, Ethical perception, Transplantation, Medical students, Willingness to donate, Decision-making, Knowledge-attitude-behaviour model, health education

Posted Date: January 31st, 2022

DOl: https://doi.org/10.21203/rs.3.rs-1281849/v1

License: (c) (1) This work is licensed under a Creative Commons Attribution 4.0 International License. Read Full License 


\section{Abstract}

Introduction: The rate of organ donation in Hong Kong is among the lowest in developed regions. Since medical students will play an important role in counselling patients for organ donation and identifying potential donors in the future, their knowledge, attitudes and action for organ donation are important. Therefore, this study aimed to understand knowledge, attitudes and actions with regard to organ donation among medical students and investigate the factors determining the knowledge and attitudes.

Methods: In this cross-sectional study, medical students in Hong Kong were invited to complete an online questionnaire that assessed their attitudes, knowledge, action of organ donation, belief and perception on death and organ donation, commitment towards organ donation, perceived confidence and competence on discussing organ donation, exposure to registered organ donors and potential organ recipients, and selfcompetence in death work. Linear regression analyses and logistic regression were performed to analyse the effect of the variables on knowledge, attitudes and action for organ donation.

Results: 377 medical students participated in the study. Almost all medical students (99.5\%) held a positive attitude towards organ donation, but only $28.1 \%$ have signed up as organ donors. Determinants of knowledge of organ donation included belief in preservation of intact body after death $(\beta=-.135,95 \% \mathrm{Cl}=$ $-.235--.036)$ and perceived confidence and competence of organ donation discussion $(\beta=-.120,95 \% \mathrm{Cl}=$ $-.221--.018)$. Predictors of organ donor registration status included knowledge of organ donation (OR = $1.031,95 \% \mathrm{Cl}=1.006-1.060)$, perceived convenience of organ donation registration $(\mathrm{OR}=3.751,95 \% \mathrm{Cl}=$ $1.616-8.710)$, commitment to organ donation $(\mathrm{OR}=3.810,95 \% \mathrm{Cl}=2.012-7.210)$, and exposure to organ donation $(\mathrm{OR}=4.283,95 \% \mathrm{Cl}=2.370-7.740)$.

Conclusions and Relevance: Knowledge is positively associated with organ donation action. The above determinants of knowledge of organ donation in medical education and health promotion could be emphasized in medical education. Future research may evaluate the effectiveness of different education programmes in improving the knowledge and actions of medical students.

\section{Introduction}

The rate of organ donation in Hong Kong has been low in the past decade (1), despite numerous recent measures by the government attempting to facilitate and coordinate the process of organ donation for example, by establishing a Committee on Promotion of Organ Donation in 2016 (2) and establishing the Centralised Organ Donation Register (CODR) allows the general population to register their wishes to donate organs after death in 2008 (3). However, in 2017, there were only 6.0 donors per million populations, which were lower than most countries adopting either opt-in or opt-out organ donation system (4). Because of the shortage of organs from both deceased and living donors, there is a long waiting time for organ transplantation in Hong Kong, for example, 53 months in average for renal failure patients and 43 months for liver failure patients in 2019 (5). This does not only increase the risk of complications but also creates adverse impacts on patients' quality of life (4). 
It is widely agreed that healthcare professionals play a significant role in organ transplantation and donation, including the identification of potential donors and the transformation of potential donors into actual donor. Previous studies showed healthcare professionals with positive attitudes towards organ donation are more likely to begin the organ donation process by seeking and obtaining consent from potential donor families (6).

Medical students are future healthcare professionals. However, there is a gap between knowledge and attitudes of organ donation to signing up as organ donors. In a Hong Kong study in 2006, although $85 \%$ of medical students showed a positive attitude towards organ donation, only $23 \%$ of them had signed the organ donation card (7). Discrepancy between knowledge, attitude, and action among medical students were also observed in China (8), Poland (9), Greece (10) and India (11). A study showed that only less than half of medical students in Hong Kong recognized brainstem death was medically and legally accepted as death (12). Lack of knowledge about organ donor might explain the low rate of action (7).

Since medical students are the future practitioners who will play a major role in organ donation counselling and medical treatment termination, it is of utmost importance to understand how their knowledge and attitude affect their action of organ donation. Previous studies also suggested that health professionals' favourable attitude toward organ donation played a role in positively determining the decision of potential donor family $(13,14)$. Identifying the determinants of action of organ donation allows interventions to promote and facilitate organ donation effects. There is a research gap in Hong Kong as the latest similar study was conducted in 2008 (7) while organ donation promotion as well as medical education have undergone dramatic changes in terms of the content of curriculum and teaching method and activities in recent years. An updated understanding in this aspect enables comparison between the action, attitude and constraints about organ donation among medical students and potentially impact the future development in organ donation promotion strategies.

\section{Methodology}

\section{Aim}

This study aims to understand knowledge, attitudes and actions with regard to organ donations among Hong Kong medical students and investigate the associated factors determining the knowledge and attitude.

\section{Setting and participants}

In this cross-sectional study, medical students in Hong Kong were recruited physically in the campuses as well as online in social media platform during December 2020 and March 2021. Only medical students currently studying in the universities in Hong Kong, i.e. The University of Hong Kong and The Chinese University of Hong Kong, were included while graduates and medical students who were taking gap year from medical study or enrolled in medical schools outside Hong Kong were excluded. All students were invited to do the self-administered English questionnaire in an online survey platform without undue pressure and no benefits were provided to students who have completed the survey. Participants were encouraged to provide their student numbers for the research team to check for duplication. Background of this study was well 
explained in the first page of the questionnaire and informed consent was obtained from all participants at the beginning of the survey. All methods were carried out in accordance with relevant guidelines and regulations

\section{Sample size estimation}

The target population is medical students from studying in Hong Kong. The population for our study is around 3000 people. At the confidence level of $95 \%$ and the margin of error as $5 \%$, the calculated sample size is that at least 341 respondents from medical students studying either in The University of Hong Kong or The Chinese University of Hong Kong are needed.

\section{Instruments}

The survey consisted of 54 items covering demographic characteristics, as well as three main outcomes which were the knowledge of organ donation, attitude and action about organ donation, and potential factors associated with organ donation. The whole set of questionnaire is attached in Appendix I.

The knowledge of organ donation was measured by 15 questions, adapted from a previous study (7). The items assessed five aspects of knowledge - organ transplantation, brain death, supply and demand of cadaveric organ, donor registration and medico-legal issues. Participants were required to provide dichotomous responses ("yes" or "no") and the score was averaged to subscale scores and a composite score. The score refers to the percentage of questions answered correctly.

Three dichotomous questions assessed the participants' attitude and action concerning organ donation, including their acceptance to organ donation, willingness to register as organ donor and action of organ donation registration.

Regarding the potential determinants influencing attitude and action of organ donation, 26 items, adapted from previous studies $(7,15,16)$, were used to assess five areas of interest - (i) belief and perception on death and organ donation (9 items), (ii) commitment towards organ donation (6 items), (iii) perceived confidence and competence on discussing organ donation (3 items), (iv) exposure to registered organ donors and potential organ recipients ( 2 items), and (v) self-competence in death work (6 items) from adaptation of Self-Competence in Death Work Scale (SC-DWS). All the questions were dichotomous except for selfcompetence in death work using five-point Likert scale where possible responses ranged from "completely incompatible" to "completely compatible" (16).

\section{Ethical consideration}

Ethics approval was granted by the Institutional Review Board (IRB) of The University of Hong Kong/Hospital Authority Hong Kong West Cluster (IRB: UW 20-748). Participation of this study was voluntary, and all the responses were kept confidential.

\section{Statistical analysis}

Demographic data were analysed by descriptive statistics. To explore the effect of clinical exposure on participant's knowledge, attitudes and action of organ donation, participants were divided into two groups for analysis: preclinical and clinical. Participants in the first, second and third academic year mainly took courses 
about anatomy, physiology and biochemistry and thus they were categorized in "preclinical" group. While for "clinical" group, students in their fourth, fifth and final year had more clinical exposure as they mainly received the teaching in the hospital and had patient contact. Linear regression analyses were conducted to analyse the effect of year of study on knowledge of organ donation. Logistic regression analysis was performed to analyse the effect of year of study on attitudes and action of organ donation, and perceived confidence and competence on discussing organ donation.

The effects of the proposed factors on knowledge of organ donation were first analysed by univariate linear regression, and subsequently by multivariable linear regression to include all the significant factors in univariate analysis and control for the effects of demographic variables, including age, gender, and university. Similarly, the effects of the proposed factors on action of organ donation were first analysed by univariate logistic regression, and subsequently by multivariable logistic regression to include all the significant factors in univariate analysis and control for the effects of demographic variables.

All statistical analyses were performed using the JAMOVI software (Version 1.6.15). All statistical tests were two sided and $\mathrm{P}$ values at the $5 \%$ level were considered statistically significant in the study.

\section{Results}

A total of 377 medical students participated in the study. The mean age was 21.0 ( $S D=2.47$ years). $51.5 \%$ of the study participants were female and $65.0 \%$ of them were preclinical students. Almost all of the respondents (99.5\%) had a positive attitude towards organ donation, and the majority (89.9\%) have considered to be a donor. However, only about one fourth of the participants $(28.1 \%)$ have signed up as a donor, showing a gap between respondents' attitudes and practice. Mean knowledge score was $67.7 \%$ (SD = $1.2 \%)$ and the self-competence in death work score was 3.22 out of $5(\mathrm{SD}=0.60)$. The demographic characteristics of the participants were reported in Table 1. 
Table 1

Descriptive statistics of main variables and socio-demographic characteristics of participants

\begin{tabular}{|ll|}
\hline Demographic Characteristics & $\mathbf{N}(\%)$ or Mean (SD) \\
\hline Gender & $\mathrm{N}=377$ \\
\hline Male & $183(48.5 \%)$ \\
\hline Age of students & $194(51.5 \%)$ \\
\hline University & $21.0(2.47)$ \\
\hline - University of Hong Kong & \\
\hline - The Chinese University of Hong Kong & $259(68.7 \%)$ \\
\hline Year of Study & $118(31.3 \%)$ \\
\hline Year 1 & \\
\hline - Year 2 & $61(16.2 \%)$ \\
\hline Y Year 3 & $142(37.7 \%)$ \\
\hline Y Year 4 & $42(11.1 \%)$ \\
\hline Y Year 5 & $61(16.2 \%)$ \\
\hline Y Year 6 & $32(8.5 \%)$ \\
\hline Attitudes - Supporting Organ Donation & $39(10.3 \%)$ \\
\hline Attitudes - Thought about Organ Donation & $339(99.5 \%)$ \\
\hline Action - Signed up as Organ Donor & $106(28.1 \%)$ \\
\hline Knowledge Score (Scale: 0-100\%) & $67.7 \%(1.2 \%)$ \\
\hline Death Competence (Scale: 1-5) & $3.22(.60)$ \\
\hline
\end{tabular}

Table 2 showed the variations of knowledge, attitudes, action and other factors along with year of study. Knowledge of organ donation differed significantly along with the year of study $(p<.001)$. Post-hoc analysis showed that students in clinical years scored significantly higher than those in preclinical years $(p<.001)$. Among the five aspects of knowledge, medical knowledge of organ transplantation, knowledge about supply and demand of cadaveric organ, and medico-legal issues differed significantly with year of study (all $p$ 's < $.05)$, although comparisons showed that students in clinical years scored significantly higher than in preclinical years only for medical knowledge $(p<.001)$. Respondents' action of becoming an organ donor also depended on their year of study $(p<.001)$. 
Table 2

Variation of Knowledge, Attitudes, Action and other factors along with Year of Study

\begin{tabular}{|c|c|c|c|c|c|c|c|c|}
\hline \multirow{2}{*}{$\begin{array}{l}\text { Aspects of } \\
\text { organ donation }\end{array}$} & \multicolumn{6}{|c|}{ Year of medical study } & \multirow{2}{*}{$\begin{array}{l}P \\
\text { value }\end{array}$} & \multirow{2}{*}{$\begin{array}{l}\text { Comparison } \\
\text { between } \\
\text { Preclinical } \\
\text { and Clinical } \\
\text { years }^{3}\end{array}$} \\
\hline & $\begin{array}{l}1 \\
(\mathrm{~N}=61)\end{array}$ & $\begin{array}{l}2 \\
(N=142)\end{array}$ & $\begin{array}{l}3 \\
(\mathrm{~N}=42)\end{array}$ & $\begin{array}{l}4 \\
(\mathrm{~N}=61)\end{array}$ & $\begin{array}{l}5 \\
(\mathrm{~N}=32)\end{array}$ & $\begin{array}{l}6 \\
(N=39)\end{array}$ & & \\
\hline $\begin{array}{l}\text { Overall } \\
\text { knowledge }\end{array}$ & $\begin{array}{l}63 \% \\
(11 \%)\end{array}$ & $\begin{array}{l}65 \% \\
(11 \%)\end{array}$ & $\begin{array}{l}67 \% \\
(11 \%)\end{array}$ & $\begin{array}{l}69 \% \\
(13 \%)\end{array}$ & $\begin{array}{l}75 \% \\
(9 \%)\end{array}$ & $\begin{array}{l}77 \% \\
(11 \%)\end{array}$ & $\begin{array}{l}\mathrm{p}< \\
.001 \star \star \star\end{array}$ & $\mathrm{p}<.001^{\star \star \star}$ \\
\hline $\begin{array}{l}\text { (1) Medical } \\
\text { knowledge of } \\
\text { organ } \\
\text { transplantation } \\
\text { (Average of } \\
\text { Q1-7) }\end{array}$ & $\begin{array}{l}57 \% \\
(18 \%)\end{array}$ & $\begin{array}{l}60 \% \\
(17 \%)\end{array}$ & $\begin{array}{l}58 \% \\
(18 \%)\end{array}$ & $\begin{array}{l}65 \% \\
(19 \%)\end{array}$ & $\begin{array}{l}74 \% \\
(15 \%)\end{array}$ & $\begin{array}{l}80 \% \\
(14 \%)\end{array}$ & $\begin{array}{l}\mathrm{p}< \\
.001 \star \star \star\end{array}$ & $\mathrm{p}<.001^{\star \star \star}$ \\
\hline $\begin{array}{l}\text { (2) Brain death } \\
\text { Average of } \\
\text { Q8-10) }\end{array}$ & $\begin{array}{l}77 \% \\
(21 \%)\end{array}$ & $\begin{array}{l}71 \% \\
(24 \%)\end{array}$ & $\begin{array}{l}78 \% \\
(23 \%)\end{array}$ & $\begin{array}{l}80 \% \\
(22 \%)\end{array}$ & $\begin{array}{l}80 \% \\
(21 \%)\end{array}$ & $\begin{array}{l}78 \% \\
(22 \%)\end{array}$ & $\begin{array}{l}p= \\
.104\end{array}$ & $p=.13$ \\
\hline $\begin{array}{l}\text { (3) Supply and } \\
\text { demand of } \\
\text { cadaveric } \\
\text { organ }(\text { Average } \\
\text { of Q11,13,14) }\end{array}$ & $\begin{array}{l}79 \% \\
(24 \%)\end{array}$ & $\begin{array}{l}82 \% \\
(23 \%)\end{array}$ & $\begin{array}{l}90 \% \\
(17 \%)\end{array}$ & $\begin{array}{l}80 \% \\
(23 \%)\end{array}$ & $\begin{array}{l}88 \% \\
(22 \%)\end{array}$ & $\begin{array}{l}89 \% \\
(18 \%)\end{array}$ & $\begin{array}{l}\mathrm{p}< \\
.001 \star \star \star\end{array}$ & $p=.48$ \\
\hline $\begin{array}{l}\text { (4) Donor } \\
\text { registration } \\
\text { (Q12) }\end{array}$ & $\begin{array}{l}30 \% \\
(46 \%)\end{array}$ & $\begin{array}{l}24 \% \\
(43 \%)\end{array}$ & $\begin{array}{l}24 \% \\
(43 \%)\end{array}$ & $\begin{array}{l}20 \% \\
(40 \%)\end{array}$ & $\begin{array}{l}41 \% \\
(50 \%)\end{array}$ & $\begin{array}{l}23 \% \\
(43 \%)\end{array}$ & $\begin{array}{l}\mathrm{p}= \\
.467\end{array}$ & $p=.69$ \\
\hline $\begin{array}{l}\text { (5) Medico- } \\
\text { legal issues } \\
\text { (Q15) }\end{array}$ & $\begin{array}{l}48 \% \\
(50 \%)\end{array}$ & $\begin{array}{l}74 \% \\
(44 \%)\end{array}$ & $\begin{array}{l}76 \% \\
(43 \%)\end{array}$ & $\begin{array}{l}77 \% \\
(42 \%)\end{array}$ & $\begin{array}{l}72 \% \\
(46 \%)\end{array}$ & $\begin{array}{l}77 \% \\
(43 \%)\end{array}$ & $\begin{array}{l}p= \\
.023^{\star}\end{array}$ & $p=.07$ \\
\hline $\begin{array}{l}\text { Positive } \\
\text { attitude and } \\
\text { behaviour }\end{array}$ & & & & & & & & \\
\hline $\begin{array}{l}\text { (1) Reporting } \\
\text { Positive } \\
\text { attitude to } \\
\text { organ donation }\end{array}$ & $98 \%$ & $99 \%$ & $100 \%$ & $100 \%$ & $100 \%$ & $100 \%$ & $\begin{array}{l}\mathrm{p}= \\
.225\end{array}$ & $p=.36$ \\
\hline
\end{tabular}

Note.

1. $P$ values of knowledge were obtained by linear regression. $P$ values of attitude, behaviour, and perceived confidence and competence were obtained by logistic regression.

2. Adjusted for age, gender, and university.

3. Preclinical years include years 1,2 and 3 whereas clinical years include years 4, 5, and 6 .

$\star p<0.05 ; * \star p<0.01 ; * \star * p<0.001$ 


\begin{tabular}{|c|c|c|c|c|c|c|c|c|}
\hline \multirow{2}{*}{$\begin{array}{l}\text { Aspects of } \\
\text { organ donation }\end{array}$} & \multicolumn{6}{|c|}{ Year of medical study } & \multirow{2}{*}{$\begin{array}{l}P \\
\text { value }\end{array}$} & \multirow{2}{*}{$\begin{array}{l}\text { Comparison } \\
\text { between } \\
\text { Preclinical } \\
\text { and Clinical } \\
\text { years }^{3}\end{array}$} \\
\hline & $\begin{array}{l}1 \\
(\mathrm{~N}=61)\end{array}$ & $\begin{array}{l}2 \\
(N=142)\end{array}$ & $\begin{array}{l}3 \\
(\mathrm{~N}=42)\end{array}$ & $\begin{array}{l}4 \\
(\mathrm{~N}=61)\end{array}$ & $\begin{array}{l}5 \\
(\mathrm{~N}=32)\end{array}$ & $\stackrel{6}{(N=39)}$ & & \\
\hline $\begin{array}{l}\text { (2) Thought } \\
\text { about organ } \\
\text { donation }\end{array}$ & $87 \%$ & $88 \%$ & $91 \%$ & $92 \%$ & $97 \%$ & $92 \%$ & $\begin{array}{l}p= \\
.068\end{array}$ & $\mathrm{p}=.14$ \\
\hline $\begin{array}{l}\text { (3) Registered } \\
\text { as organ donor }\end{array}$ & $13 \%$ & $23 \%$ & $21 \%$ & $33 \%$ & $50 \%$ & $51 \%$ & $\begin{array}{l}\mathrm{p}< \\
.001 \star \star \star\end{array}$ & $\mathrm{p}<.001^{\star \star \star}$ \\
\hline \multicolumn{9}{|l|}{$\begin{array}{l}\text { Perceived } \\
\text { confidence and } \\
\text { competence on } \\
\text { discussing } \\
\text { organ } \\
\text { donation. }\end{array}$} \\
\hline $\begin{array}{l}\text { (1) Feeling } \\
\text { uncomfortable } \\
\text { to think or talk } \\
\text { about organ } \\
\text { donation }\end{array}$ & $7 \%$ & $11 \%$ & $0 \%$ & $13 \%$ & $6 \%$ & $13 \%$ & $\begin{array}{l}\mathrm{p}= \\
.923\end{array}$ & $p=.13$ \\
\hline $\begin{array}{l}\text { (2) Perceiving } \\
\text { themselves as } \\
\text { Competent in } \\
\text { organ donation } \\
\text { counselling }\end{array}$ & $39 \%$ & $40 \%$ & $29 \%$ & $41 \%$ & $38 \%$ & $39 \%$ & $\begin{array}{l}p= \\
.302\end{array}$ & $p=.60$ \\
\hline $\begin{array}{l}\text { (3) Perceiving } \\
\text { themselves as } \\
\text { Confident in } \\
\text { organ donation } \\
\text { counselling }\end{array}$ & $44 \%$ & $42 \%$ & $29 \%$ & $33 \%$ & $28 \%$ & $23 \%$ & $\begin{array}{l}p= \\
.004^{\star *}\end{array}$ & $p=.06$ \\
\hline \multicolumn{9}{|l|}{ Note. } \\
\hline \multicolumn{9}{|c|}{$\begin{array}{l}\text { 1. } P \text { values of knowledge were obtained by linear regression. } P \text { values of attitude, behaviour, and } \\
\text { perceived confidence and competence were obtained by logistic regression. }\end{array}$} \\
\hline \multicolumn{9}{|c|}{ 2. Adjusted for age, gender, and university. } \\
\hline \multicolumn{9}{|c|}{ 3. Preclinical years include years 1,2 and 3 whereas clinical years include years 4,5 , and 6 . } \\
\hline$\star p<0.05 ; * \star p<$ & $1 ; * \star \star p$ & 001. & & & & & & \\
\hline
\end{tabular}

Table 3 demonstrated the effect of the proposed factors on knowledge of organ donation using linear regression. Two factors (believing the preservation of an intact body after death as important, and approaching family members of potential donors) remained significant associated with higher knowledge scores after adjustment for demographic variables (age, gender, and university). 
Table 3

Linear Regression analysis predicting Knowledge of organ donation with the proposed factors

\begin{tabular}{|c|c|c|c|c|c|c|c|}
\hline & & \multicolumn{3}{|c|}{ Univariate analysis } & \multicolumn{3}{|c|}{ Multivariable analysis } \\
\hline & & Estimate & $95 \% \mathrm{Cl}$ & $\begin{array}{l}\mathrm{P} \\
\text { value }^{1}\end{array}$ & Estimate & $95 \% \mathrm{Cl}$ & $\begin{array}{l}\mathrm{P} \\
\text { value }^{2}\end{array}$ \\
\hline \multirow[t]{8}{*}{ (1) Belief } & $\begin{array}{l}\text { I think the } \\
\text { preservation of an } \\
\text { intact body after } \\
\text { death is important. }\end{array}$ & -0.164 & $\begin{array}{l}(-.260 \\
-.060)\end{array}$ & $.001^{\star \star}$ & -0.135 & $\begin{array}{l}(-.235 \\
-.036)\end{array}$ & $.008^{\star \star}$ \\
\hline & $\begin{array}{l}\text { I think the body will } \\
\text { be disfigured when } \\
\text { the organs are } \\
\text { removed. }\end{array}$ & -0.042 & $\begin{array}{l}(-0.143, \\
-0.060)\end{array}$ & 0.421 & & & \\
\hline & $\begin{array}{l}\text { I think donating } \\
\text { one's organs adds } \\
\text { meaning to one's } \\
\text { life. }\end{array}$ & -0.001 & $\begin{array}{l}(-0.113 \\
0.090)\end{array}$ & 0.817 & & & \\
\hline & $\begin{array}{l}\text { I think live organ } \\
\text { donation is better } \\
\text { than cadaveric } \\
\text { organ donation in } \\
\text { solving the problem } \\
\text { of organ shortage. }\end{array}$ & -0.085 & $\begin{array}{l}(-0.187 \\
0.016)\end{array}$ & 0.098 & & & \\
\hline & $\begin{array}{l}\text { I think donating } \\
\text { organs is an action } \\
\text { to save lives. }\end{array}$ & -0.045 & $\begin{array}{l}(-0.151 \\
0.052)\end{array}$ & 0.335 & & & \\
\hline & $\begin{array}{l}\text { My family would } \\
\text { object if I were to } \\
\text { donate my organs. }\end{array}$ & -0.028 & $\begin{array}{l}(-0.129 \\
0.074)\end{array}$ & 0.594 & & & \\
\hline & $\begin{array}{l}\text { I think there will be } \\
\text { premature } \\
\text { termination of } \\
\text { medical treatment } \\
\text { for registered organ } \\
\text { donors. }\end{array}$ & -0.125 & $\begin{array}{l}(-0.225 \\
-0.024)\end{array}$ & $0.016^{*}$ & -0.088 & $\begin{array}{l}(-0.190 \\
0.014)\end{array}$ & 0.091 \\
\hline & $\begin{array}{l}\text { I think it is } \\
\text { convenient to } \\
\text { register as an organ } \\
\text { donor in Hong } \\
\text { Kong. }\end{array}$ & 0.025 & $\begin{array}{l}(-0.077 \\
0.126)\end{array}$ & 0.63 & & & \\
\hline
\end{tabular}

Note:

${ }^{1} \mathrm{P}$ values of univariate analysis were obtained by linear regression.

${ }^{2} \mathrm{P}$ values of multivariable analysis were obtained by linear regression including all the significant factors in the univariate analysis and controlled for age, gender, and university.

$\star p<0.05 ; * \star p<0.01 ; * \star \star p<0.001$ 
I think the

promotion and

information of

organ donation in

Hong Kong is

sufficient.

\section{(2)}

Commitment

I will agree to the

donation of my

family members'

organs.

$$
-0.065
$$

$$
(-0.166
$$

0.21

$$
0.037)
$$

0.100

$(-0.000$,

0.052

$0.201)$
(3) Perceived

\begin{tabular}{|c|c|c|}
\hline $\begin{array}{l}\text { I have discussed the } \\
\text { organ donation with } \\
\text { my family. }\end{array}$ & 0.043 & $\begin{array}{l}(-0.0581 \\
0.145)\end{array}$ \\
\hline $\begin{array}{l}\text { I have received } \\
\text { training in how to } \\
\text { request organ } \\
\text { donation in } \\
\text { potential donors or } \\
\text { their family } \\
\text { members. }\end{array}$ & -0.073 & $\begin{array}{l}(-0.175 \\
0.028)\end{array}$ \\
\hline
\end{tabular}
confidence and competence
0.107

(0.016)

0.208 )

$0.038^{*}$

0.095

$(-0.005$,

$0.195)$

0.063

register as an organ

donor.

$\begin{array}{llll}\text { I am a regular blood } & 0.031 & (-0.070, & 0.548 \\ \text { donor. } & & 0.133) & \end{array}$

I volunteer regularly.

$-0.113$

$(-0.214$,

$0.143 \quad-0.0752$

$(-0.176$, $0.026)$

0.143

-0.012 )

I feel uncomfortable to think or talk

$(-0.158$,

0.27

about organ

$-0.057$

$0.044)$

donation.

I am confident in counseling patients $-0.084$

$(-0.185$

0.103

regarding the issues

$0.017)$

of organ donation.

Note:

${ }^{1} \mathrm{P}$ values of univariate analysis were obtained by linear regression.

${ }^{2} \mathrm{P}$ values of multivariable analysis were obtained by linear regression including all the significant factors in the univariate analysis and controlled for age, gender, and university.

$* p<0.05 ; * \star p<0.01 ; * \star \star p<0.001$ 


\begin{tabular}{|c|c|c|c|c|c|c|c|}
\hline & \multirow[b]{2}{*}{$\begin{array}{l}\text { I am confident in } \\
\text { approaching family } \\
\text { members of } \\
\text { potential donors } \\
\text { diagnosed brain } \\
\text { death and } \\
\text { discussing issued } \\
\text { related to organ } \\
\text { donation. }\end{array}$} & \multicolumn{3}{|c|}{ Univariate analysis } & \multicolumn{3}{|c|}{ Multivariable analysis } \\
\hline & & -0.154 & $\begin{array}{c}(-0.255 \\
-0.054)\end{array}$ & $0.003^{\star \star}$ & -0.1198 & $\begin{array}{l}(-0.221 \\
-0.018)\end{array}$ & $0.022^{\star}$ \\
\hline \multirow[t]{2}{*}{ (4) Exposure } & $\begin{array}{l}\text { I know family } \\
\text { members or close } \\
\text { friends who have } \\
\text { registered as an } \\
\text { organ donor. }\end{array}$ & 0.098 & $\begin{array}{l}(-0.003, \\
0.199)\end{array}$ & 0.057 & & & \\
\hline & $\begin{array}{l}\text { I know people who } \\
\text { have benefited or } \\
\text { are in need of an } \\
\text { organ transplant. }\end{array}$ & -0.090 & $\begin{array}{l}(-0.191 \\
0.013)\end{array}$ & 0.082 & & & \\
\hline $\begin{array}{l}\text { (5) Death } \\
\text { competence }\end{array}$ & $\begin{array}{l}\text { Self-Competence in } \\
\text { Death Work Scale }\end{array}$ & .010 & $\begin{array}{l}(-.060 \\
.140)\end{array}$ & 0.497 & & & \\
\hline \multicolumn{8}{|l|}{ Note: } \\
\hline \multicolumn{8}{|c|}{${ }^{1} \mathrm{P}$ values of univariate analysis were obtained by linear regression. } \\
\hline \multicolumn{8}{|c|}{$\begin{array}{l}{ }^{2} \mathrm{P} \text { values of multivariable analysis were obtained by linear regression including all the significant factors } \\
\text { in the univariate analysis and controlled for age, gender, and university. }\end{array}$} \\
\hline$\star p<0.05 ; * \star$ & $0.01 ; * \star \star p<0.001$ & & & & & & \\
\hline
\end{tabular}

Table 4 demonstrated the effect of the proposed factors on the registration status as organ donor using logistic regression. Four factors (belief of finding it convenient to register, two items of commitment to organ donation were also associated with higher action in the model, specifically, having discussed organ donation with their family, and having regular blood donation, and knowledge of organ donation) remained significant associated with higher registration status as organ donor after adjustment for demographic variables (age, gender, and university). 
Table 4

Logistic Regression analysis predicting Action of organ donation with the proposed factors

\begin{tabular}{|c|c|c|c|c|c|}
\hline & & \multicolumn{2}{|c|}{ Univariate analysis } & \multicolumn{2}{|c|}{$\begin{array}{l}\text { Multivariable } \\
\text { analysis }\end{array}$} \\
\hline & & $\begin{array}{l}\text { Odds ratio, } \\
95 \% \mathrm{Cl}\end{array}$ & $P$ value $^{1}$ & $\begin{array}{l}\text { Odds } \\
\text { ratio, } \\
95 \% \mathrm{Cl}\end{array}$ & Pvalue $^{2}$ \\
\hline \multirow[t]{15}{*}{ (1) Belief } & \multirow{2}{*}{$\begin{array}{l}\text { I think the preservation of an intact } \\
\text { body after death is important. }\end{array}$} & 0.683 & .218 & & \\
\hline & & $\begin{array}{l}(0.372, \\
1.253)\end{array}$ & & & \\
\hline & \multirow{2}{*}{$\begin{array}{l}\text { I think the body will be disfigured } \\
\text { when the organs are removed. }\end{array}$} & 1.01 & 0.978 & & \\
\hline & & $\begin{array}{l}(0.589, \\
1.724)\end{array}$ & & & \\
\hline & \multirow{2}{*}{$\begin{array}{l}\text { I think donating one's organs adds } \\
\text { meaning to one's life. }\end{array}$} & 1.70 & 0.301 & & \\
\hline & & $\begin{array}{l}(0.623, \\
4.623)\end{array}$ & & & \\
\hline & \multirow{2}{*}{$\begin{array}{l}\text { I think live organ donation is better } \\
\text { than cadaveric organ donation in } \\
\text { solving the problem of organ } \\
\text { shortage. }\end{array}$} & 0.875 & 0.575 & & \\
\hline & & $\begin{array}{l}(0.547 \\
1.398)\end{array}$ & & & \\
\hline & $\begin{array}{l}\text { I think donating organs is an action } \\
\text { to save lives. }\end{array}$ & $\begin{array}{l}834673.15 \\
(0.00, \text { inf })\end{array}$ & 0.983 & & \\
\hline & \multirow{2}{*}{$\begin{array}{l}\text { My family would object if I were to } \\
\text { donate my organs. }\end{array}$} & 0.520 & $0.032^{\star}$ & 0.770 & 0.521 \\
\hline & & $\begin{array}{l}(0.286, \\
0.946)\end{array}$ & & $\begin{array}{l}(0.353, \\
1.680)\end{array}$ & \\
\hline & \multirow{2}{*}{$\begin{array}{l}\text { I think there will be premature } \\
\text { termination of medical treatment for } \\
\text { registered organ donors. }\end{array}$} & 1.159 & 0.697 & & \\
\hline & & $\begin{array}{l}(0.561, \\
2.371)^{\prime}\end{array}$ & & & \\
\hline & \multirow{2}{*}{$\begin{array}{l}\text { I think it is convenient to register as } \\
\text { an organ donor in Hong Kong. }\end{array}$} & 6.612 & $<0.001^{\star * \star}$ & 3.751 & $0.002^{\star \star}$ \\
\hline & & $\begin{array}{l}(3.084 \\
14.176)\end{array}$ & & $\begin{array}{l}(1.616 \\
8.710)\end{array}$ & \\
\hline
\end{tabular}

Note:

${ }^{1} \mathrm{P}$ values of univariate analysis were obtained by logistic regression.

${ }^{2} \mathrm{P}$ values of multivariable analysis were obtained by logistic regression including all the significant factors in the univariate analysis and controlled for age, gender, and university.

${ }^{3}$ Medical knowledge of organ transplantation was not included in the multivariable model because of significant collinearity with overall Knowledge of Organ donation.

$* p<0.05 ; * \star p<0.01 ; * \star \star p<0.001$ 


\begin{tabular}{|c|c|c|c|c|c|}
\hline & & \multicolumn{2}{|c|}{ Univariate analysis } & \multicolumn{2}{|c|}{$\begin{array}{l}\text { Multivariable } \\
\text { analysis }\end{array}$} \\
\hline & \multirow{2}{*}{$\begin{array}{l}\text { I think the promotion and } \\
\text { information of organ donation in } \\
\text { Hong Kong is sufficient. }\end{array}$} & 0.861 & 0.566 & & \\
\hline & & $\begin{array}{l}(0.517 \\
1.435)\end{array}$ & & & \\
\hline \multirow{10}{*}{$\begin{array}{l}\text { (2) } \\
\text { Commitment }\end{array}$} & \multirow{2}{*}{$\begin{array}{l}\text { I will agree to the donation of my } \\
\text { family members' organs. }\end{array}$} & 3.048 & $0.005^{\star \star}$ & 2.234 & 0.090 \\
\hline & & $\begin{array}{l}(1.398 \\
6.649)\end{array}$ & & $\begin{array}{l}(0.881, \\
5.560)^{\prime}\end{array}$ & \\
\hline & \multirow{2}{*}{$\begin{array}{l}\text { I have discussed the organ donation } \\
\text { with my family. }\end{array}$} & 4.797 & $<0.001^{\star \star \star}$ & 3.083 & $<0.001^{\star \star \star}$ \\
\hline & & $\begin{array}{l}(2.960 \\
7.773)\end{array}$ & & $\begin{array}{l}(1.748, \\
5.440)\end{array}$ & \\
\hline & \multirow{2}{*}{$\begin{array}{l}\text { I have received training in how to } \\
\text { request organ donation in potential } \\
\text { donors or their family members. }\end{array}$} & 2.089 & 0.058 & & \\
\hline & & $\begin{array}{l}(0.977 \\
4.467)\end{array}$ & & & \\
\hline & \multirow[t]{2}{*}{ I am a regular blood donor. } & 4.574 & $<0.001^{\star \star \star}$ & 3.810 & $<0.001^{\star \star \star}$ \\
\hline & & $\begin{array}{l}(2.693, \\
7.770)\end{array}$ & & $\begin{array}{l}(2.012, \\
7.210)\end{array}$ & \\
\hline & \multirow[t]{2}{*}{ I volunteer regularly. } & 1.398 & 0.152 & & \\
\hline & & $\begin{array}{l}(0.884, \\
2.209)\end{array}$ & & & \\
\hline \multirow{4}{*}{$\begin{array}{l}\text { (3) Perceived } \\
\text { confidence } \\
\text { and } \\
\text { competence }\end{array}$} & \multirow{2}{*}{$\begin{array}{l}\text { I feel uncomfortable to think or talk } \\
\text { about organ donation. }\end{array}$} & 0.639 & 0.309 & & \\
\hline & & $\begin{array}{l}(0.269 \\
1.515)\end{array}$ & & & \\
\hline & \multirow{2}{*}{$\begin{array}{l}\text { I am confident in counseling patients } \\
\text { regarding the issues of organ } \\
\text { donation. }\end{array}$} & 1.131 & 0.599 & & \\
\hline & & $\begin{array}{l}(0.715, \\
1.790)\end{array}$ & & & \\
\hline
\end{tabular}

Note:

${ }^{1} \mathrm{P}$ values of univariate analysis were obtained by logistic regression.

${ }^{2} \mathrm{P}$ values of multivariable analysis were obtained by logistic regression including all the significant factors in the univariate analysis and controlled for age, gender, and university.

${ }^{3}$ Medical knowledge of organ transplantation was not included in the multivariable model because of significant collinearity with overall Knowledge of Organ donation.

$* p<0.05 ; * \star p<0.01 ; * \star * p<0.001$ 


\begin{tabular}{|c|c|c|c|c|c|}
\hline & & \multicolumn{2}{|c|}{ Univariate analysis } & \multicolumn{2}{|c|}{$\begin{array}{l}\text { Multivariable } \\
\text { analysis }\end{array}$} \\
\hline & \multirow{2}{*}{$\begin{array}{l}\text { I am confident in approaching family } \\
\text { members of potential donors } \\
\text { diagnosed brain death and } \\
\text { discussing issued related to organ } \\
\text { donation. }\end{array}$} & 1.028 & 0.909 & & \\
\hline & & $\begin{array}{l}(0.645, \\
1.638)\end{array}$ & & & \\
\hline \multirow[t]{4}{*}{ (4) Exposure } & \multirow{2}{*}{$\begin{array}{l}\text { I know family members or close } \\
\text { friends who have registered as an } \\
\text { organ donor. }\end{array}$} & 6.626 & $<0.001^{\star \star \star}$ & 4.283 & $<0.001^{\star \star \star \star}$ \\
\hline & & $\begin{array}{l}(3.960 \\
11.090)\end{array}$ & & $\begin{array}{l}(2.370, \\
7.740)\end{array}$ & \\
\hline & \multirow{2}{*}{$\begin{array}{l}\text { I know people who have benefited or } \\
\text { are in need of an organ transplant. }\end{array}$} & 1.369 & 0.251 & & \\
\hline & & $\begin{array}{l}(0.801 \\
2.339)\end{array}$ & & & \\
\hline \multirow{2}{*}{$\begin{array}{l}\text { (5) Death } \\
\text { competence }\end{array}$} & \multirow{2}{*}{$\begin{array}{l}\text { Self-Competence in Death Work } \\
\text { Scale }\end{array}$} & 1.556 & $.024^{\star}$ & 1.166 & 0.538 \\
\hline & & $\begin{array}{l}(1.060 \\
2.283)\end{array}$ & & $\begin{array}{l}(0.715, \\
1.900)\end{array}$ & \\
\hline \multirow{8}{*}{$\begin{array}{l}\text { (6) } \\
\text { Knowledge }\end{array}$} & \multirow{2}{*}{$\begin{array}{l}\text { Overall Knowledge of Organ } \\
\text { donation }\end{array}$} & 20.1 & $0.002^{\star \star}$ & 1.031 & $0.013^{*}$ \\
\hline & & $\begin{array}{l}(2.950 \\
138)\end{array}$ & & $\begin{array}{l}(1.006, \\
1.060)\end{array}$ & \\
\hline & \multirow{2}{*}{$\begin{array}{l}\text { Medical knowledge of organ } \\
\text { transplantation }\end{array}$} & 7.234 & $0.002 \star \star 3$ & & \\
\hline & & $\begin{array}{l}(2.050 \\
25.468)\end{array}$ & & & \\
\hline & \multirow[t]{2}{*}{ Brain death } & 2.504 & 0.082 & & \\
\hline & & $\begin{array}{l}(0.891 \\
7.036)\end{array}$ & & & \\
\hline & \multirow{2}{*}{$\begin{array}{l}\text { Supply and demand of cadaveric } \\
\text { organ }\end{array}$} & 0.712 & 0.506 & & \\
\hline & & $\begin{array}{l}(0.262, \\
1.930)\end{array}$ & & & \\
\hline
\end{tabular}

Note:

${ }^{1} \mathrm{P}$ values of univariate analysis were obtained by logistic regression.

${ }^{2} \mathrm{P}$ values of multivariable analysis were obtained by logistic regression including all the significant factors in the univariate analysis and controlled for age, gender, and university.

${ }^{3}$ Medical knowledge of organ transplantation was not included in the multivariable model because of significant collinearity with overall Knowledge of Organ donation.

$* p<0.05 ; * \star p<0.01 ; * \star * p<0.001$ 


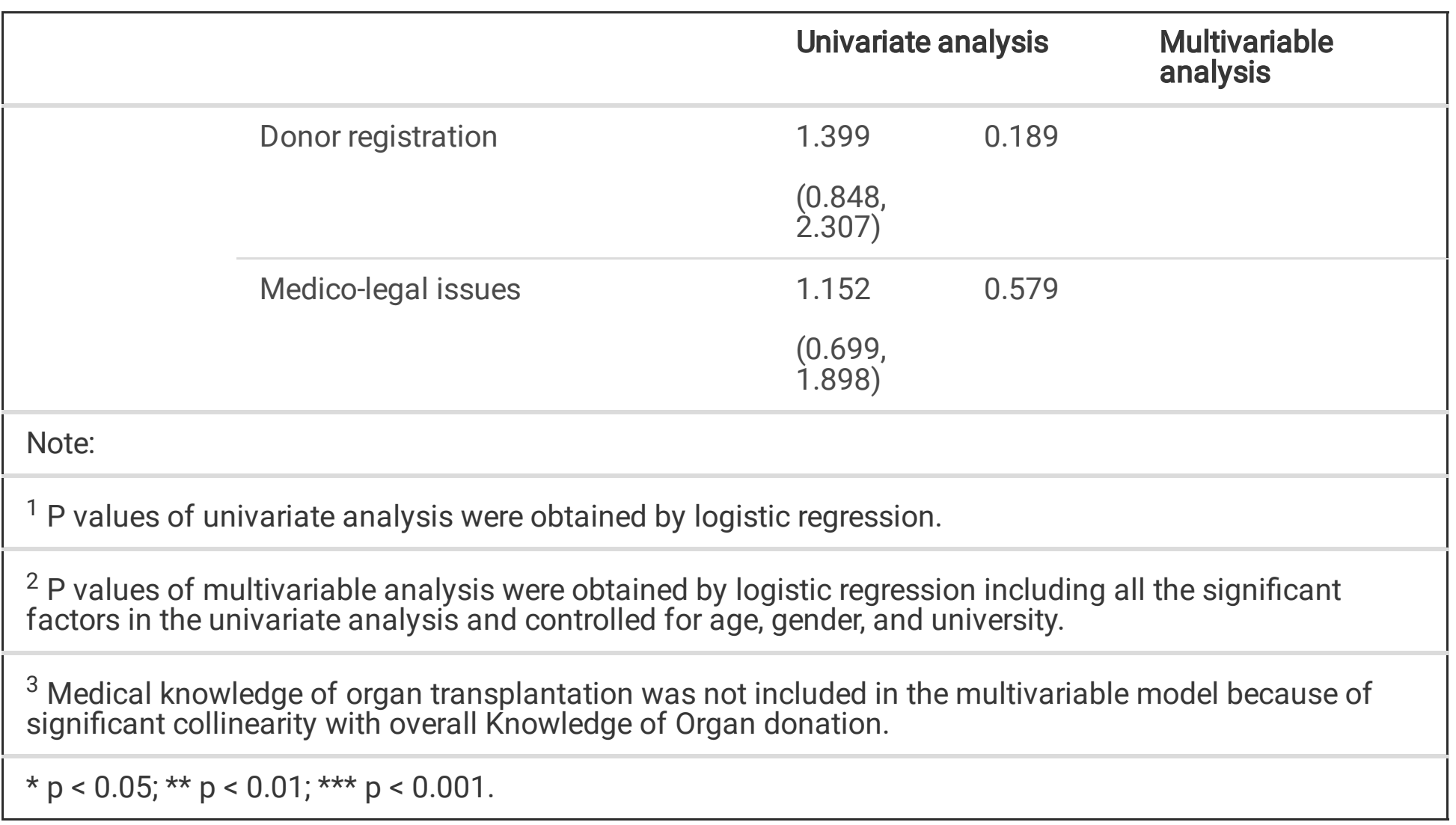

\section{Discussion}

In our study, almost all medical students held a positive attitude towards organ donation, but only about a quarter of them have signed up as organ donors. Determinants of knowledge of organ donation included belief in preservation of intact body after death and perceived confidence and competence of organ donation discussion. Predictors of registration for being an organ donor included knowledge of organ donation, perceived convenience of organ donation registration, commitment to organ donation, and exposure to organ donation.

Comparing our study with one conducted among medical students in 2008 (7), both showed very high percentage of medical students, $99.5 \%$ in our study and $99 \%$ in 2008 study respectively, supported organ donation. Percentage of medical students having the thought of registering organ donation increased from $85-89.9 \%$ and signing up as organ donors also raised from $23-28.1 \%$ from 2008 to 2021 . There are rising trends of their attitudes and actions towards organ donation over the decade, which may be related to the information promotion and convenience of registration. However, there was no substantial improvement and the gap in between knowledge and actions in organ donation needs to be addressed. Discrepancy between knowledge and action among medical students were also observed in China (8), Poland (9), Greece (10) and India (11). Previous observational and interventional studies suggested that exposure and understanding about organ transplantation process and more organ donation dialogue can increase the rate of willingness to donate $(10,17)$.

Regarding the relationship between knowledge and action, our finding that higher knowledge is associated with higher likelihood of registering as organ donor in medical students is consistent with the findings from 
the literature in general $(7,14,18)$. A Germany study of 1136 medical students showed a significant association between organ donation knowledge and likelihood of holding an organ donor card regardless of age and gender in multivariate analyses while a US study also demonstrated people with higher educational level usually had a more positive attitude as an organ donor (19). Our results adjusted with several demographic factors add more reliable evidence in the literature.

Other than knowledge, family plays an important factor in action of organ donation of medical students. Medical students with greater exposure to organ donation, for example students who have family members registered as an organ donor or who have discussed with their family about organ donation are more likely to sign the organ donation card compared to those who have no registered family members registered as donor or have not discussed with family. This findings are consistent with previous studies $(20,21)$. The importance of family in relation to organ donation may be attributed to several reasons. First, it may be related to a subjective norm, in which students think that by donating their organs, their family who did the same will give them approval. Second, it may be due to the role of family in providing information about organ donation, facilitating their signing of organ donation (21). Moreover, there is a negative association between anticipated family objection and the action of signing organ donation cards, which is consistent with the study in 2008 (7). Family influence has consistently been an important factor towards organ donation throughout the years (22) and that family support plays a great part in students' action of signing the organ donation card.

In addition, being a regular blood donor is associated with higher rate of being an organ donor. It is likely that this is because students who are regular blood donors feel more obliged to help people in need. Another possible reason is that students who have prior experience of blood donation may have a higher chance to be targeted for organ donation promotion. Our result is consistent with previous studies (8) which showed that Chinese university students who had previously donated blood demonstrated better knowledge about transplantation and also much more willing to donate their organs with an odds ratio of 1.91 .

It is important to note that respondents with higher self-perceived confidence and competence had better knowledge and higher rate of action of organ donation. Knowledge may affect people's confidence and competence, or vice versa. Confidence and competence may determine people's learning attitude, and these two factors reflect underlying qualities of students and further affect their knowledge and action.

In regard of beliefs about organ donation, our study found the belief of preservation of intact body remained important in determining knowledge of organ donation, which showed no change compared with the Hong Kong study in 2008 (7). Participants holding such belief is associated with lower knowledge score, but not associated with the rate of action. In addition, the perceived convenience of organ donation registration determined likelihood of registering as organ donors. Providing students more information about the procedures and criteria of organ donation may enhance students' motivation of organ donation registration. Therefore, these beliefs are particularly important if we were to promote organ donation to medical students.

\section{Strengths and limitations of the study}

Page 16/19 
The strengths of the present study include the additional variables tested and the statistical methods that controlled for covariates. The present study tested the effects of new variables, including prosocial activities and exposure to organ donation, on knowledge and action of organ donation among medical students. Secondly, the present study used logistic regression analysis that controlled for confounds that could affect the results, which is superior to the bivariate correlation in most previous studies. Therefore, the results from the present findings are more reliable than previous studies.

The questionnaire could have been improved by performing a pilot study for validation. There may be social desirability bias in our study which led to more students giving answers supporting organ transplantation, therefore our study may have overestimated the actual number of students who support organ transplantation.

\section{Conclusions And Recommendations}

Our study showed significant positive association between knowledge and action of organ donation, suggesting that enhancing students' knowledge can improve the percentage of organ donation registration. Since the determinants of knowledge are beliefs about organ donation, perceived confidence and competence of organ donation, medical curriculum in the universities may target on enhancing medical students' confidence and competence on organ donation discussion, and improving their knowledge on organ transplantation. Future studies may evaluate the effectiveness of different education and promotion programmes in improving medical students' knowledge and action.

\section{Declarations}

- Ethics approval and consent to participate: Ethics approval was granted by the Institutional Review Board (IRB) of The University of Hong Kong/Hospital Authority Hong Kong West Cluster (IRB: UW 20748).

- Consent for publication: not applicable

- Availability of data and materials: The datasets used and analysed during the current study are available from the corresponding author on reasonable request.

- Competing interests: not applicable

- Funding: not applicable

- Authors' contributions: Chu and Chung contributed to the conception of the work. Sin, Law, Yan prepared the questionnaire. Chu, Lam, Choi analyzed and interpreted the data. Chu, Chung, and Hui are major contributors in writing the manuscript. Wan supervised the project. All authors read and approved the final manuscript.

- Acknowledgements: not applicable

\section{References}


1. Statistics - Number of organ/tissue donation \& patient waiting for transplantation under Hong Kong Hospital Authority (1.1.2011 - 31.12.2020) Hong Kong: Department of Health; 2020 [19-04-2021]. Available from: https://www.organdonation.gov.hk/eng/statistics.html.

2. Organ Donation Promotion Charter: Department of Health; 2020 [19-04-2021]. Available from: https://www.organdonation.gov.hk/eng/charter.html.

3. Know More about Centralised Organ Donation Register Hong Kong: Department of Health 2020 [19-042021]. Available from: https://www.organdonation.gov.hk/eng/knowmore.html.

4. Teoh JYC, Lau BSY, Far NY, Yuen SKK, Yee CH, Hou SSM, et al. Attitudes, acceptance, and registration in relation to organ donation in Hong Kong: a cross-sectional study. Hong Kong Medical Journal. 2020;26(3):192-200.

5. LCQ4: Organ donation and transplants [press release]. Hong Kong: Legislative Council, 04-12-2019 2019.

6. Jawoniyi O, Gormley K, McGleenan E, Noble HR. Organ donation and transplantation: Awareness and roles of healthcare professionals - A systematic literature review. Journal of Clinical Nursing. 2018;27(56):E726-E38.

7. Chung CK, Ng CW, Li JY, Sum KC, Man AH, Chan SP, et al. Attitudes, knowledge, and actions with regard to organ donation among Hong Kong medical students. Hong Kong Med J. 2008;14(4):278-85.

8. Chen JX, Zhang TM, Lim FL, Wu HC, Lei TF, Yeong PK, et al. Current knowledge and attitudes about organ donation and transplantation among Chinese university students. 2006;38:2761-5.

9. Perenc L, Radochonski M, Radochonski A. Knowledge and attitudes of Polish university students toward organ donation and transplantation. Psychology Health \& Medicine. 2012;17(6):667-73.

10. Dardavessis T, Xenophontos P, Haidich AB, Kiritsi M, Vayionas MA. Knowledge, Attitudes and Proposals of Medical Students Concerning Transplantations in Greece. International Journal of Preventive Medicine. 2011;2(3):164-9.

11. Lavanya V, Vadapalli K, Krishnamurthy PS, Ds FNB. Comparison of knowledge, attitude about organ donation among first year medical and engineering students. Indian Journal of Clinical Anatomy and Physiology. 2019;6(4):408-14.

12. Connie FOY, Kelvin LKH, Chung AC, Diana CMK, Gilberto LKK. Knowledge, acceptance and perception towards brainstem death among medical students in Hong Kong: a questionnaire survey on brainstem death. Medical Teacher. 2008;30(5):E125-E30.

13. Bartucci MR. Organ donation: a study of the donor family perspective. The Journal of neuroscience nursing: journal of the American Association of Neuroscience Nurses. 1987;19(6):305-9.

14. Schaeffner ES, Windisch W, Freidel K, Breitenfeldt K, Winkelmayer WC. Knowledge and attitude regarding organ donation among medical students and physicians. Transplantation. 2004;77(11):1714-8.

15. Tam WWS, Suen LKP, Chan HYL. Knowledge, Attitudes and Commitment Toward Organ Donation Among Nursing Students in Hong Kong. Transplantation Proceedings. 2012;44(5):1196-200.

16. Cheung JTK, Au DWH, Chan WCH, Chan JHY, Ng K, Woo J. Self-competence in death work among health and social care workers: a region-wide survey in Hong Kong. Bmc Palliative Care. 2018;17.

17. Radunz S, Benko T, Stern S, Saner FH, Paul A, Kaiser GM. Medical students' education on organ donation and its evaluation during six consecutive years: results of a voluntary, anonymous educational 
intervention study. European Journal of Medical Research. 2015;20.

18. Ali NF, Qureshi A, Jilani BN, Zehra N. Knowledge and ethical perception regarding organ donation among medical students. Bmc Medical Ethics. 2013;14.

19. Boulware LE, Ratner LE, Sosa JA, Cooper LA, LaVeist TA, Powe NR. Determinants of willingness to donate living related and cadaveric organs: Identifying opportunities for intervention. Transplantation. 2002;73(10):1683-91.

20. Rios A, Lopez-Navas A, Lopez-Lopez A, Gomez FJ, Iriarte J, Herruzo R, et al. A Multicentre and stratified study of the attitude of medical students towards organ donation in Spain. Ethn Health. 2019;24(4):44361.

21. Guadagnoli E, Christiansen C, Dejong W, Mcnamara P, Beasley C, Christiansen E, et al. The public's willingness to discuss their preference for organ donation with family members. Clinical Transplantation. 1999;13(4):342-8.

22. Mekahli D, Liutkus A, Fargue S, Ranchin B, Cochat P, editors. Survey of first-year medical students to assess their knowledge and attitudes toward organ transplantation and donation2009: Elsevier.

\section{Supplementary Files}

This is a list of supplementary files associated with this preprint. Click to download.

- HRPPaperBMCMedicalEthics3Appendix.docx 